\title{
Analyse der Verteilung der possessiven Attribute in der deutschen Alltagssprache des 19. Jahrhunderts
}

doi.org/10.14232/jp.agi.2022.3.5

BETREUER: DR. PÉTER KAPPEL

\section{Einleitung}

Die deutsche Sprache ist regional gegliedert. Neben dem Standarddeutschen existieren moderne Regionalsprachen (Dialekte und Regiolekte), die ein Großteil der Sprecher des Deutschen auch im 21. Jh. aktiv beherrscht oder zumindest versteht (vgl. dazu Schmidt 2017: 114-129). Im Atlas zur deutschen Alltagssprache (http://www.atlas-alltagssprache.de/, im Weiteren AdA) von Elspaß und Möller (2003ff.) werden die regionalen Unterschiede der Alltagssprache visualisiert. Der AdA konzentriert sich auf den aktuellen regionalsprachlichen Wortschatz, daneben werden aber auch Aussprachevarianten und grammatische Varianten wie etwa unterschiedliche Formen der possessiven Attribute erfasst. Da ich Deutsch und Geschichte auf Lehramt studiere und mich auch sehr für Sprachgeschichte interessiere, wendete ich mich der geschichtlichen Dimension der regionalen Vielfalt zu. In der vorliegenden Arbeit soll die regionale Verteilung der verschiedenen Varianten des possessiven Attributs im 19. Jahrhundert untersucht werden. Dazu liegen keine quantitativen Untersuchungen vor, deshalb habe ich mich dafür entschieden, dieses Phänomen zu untersuchen, um zur Schließung dieser Forschungslücke beizutragen. 
Elspaß (2005: 6f.) kritisiert die traditionelle Sprachgeschichte, weil sie kaum alltagsprachliche Texte untersucht und somit einen großen Teil der Sprachrealität aus den Augen verliert. Die vorliegende Arbeit schließt sich an den Ansatz für eine ,Sprachgeschichte von unten' an und stellt das geschriebene Alltagsdeutsch in den Fokus (Elspaß 2005: 20): „Prototyp für sprachliches Handeln im sozialen Gefüge und Ausgangspunkt für sprachliche Variation und sprachlichen Wandel ist die dialogische Alltagssprache im privaten Nähebereich." Die authentische und ,natürliche' historische Alltagssprache ist nach Elspaß (2005: 20) „nur in schriftlich vermittelter Form greifbar - am ehesten in privaten Briefen von eben jenen Schreiberinnen und Schreibern, deren Sprache am wenigsten an der Schriftsprache ihrer Zeit geschult war". Die vorliegende Arbeit konzentriert sich auf das 19. Jahrhundert, weil aus dieser Zeit viele von Historikern gesammelte Quellen dieser Art zur Verfügung stehen (vgl. Elspaß 2005: 11) und sie einen neuen Blick in die regionale und diachrone Variation der possessiven Attribute ermöglichen. Die bisherigen Untersuchungsergebnisse zu den Genitivattributen (vgl. z.B. Admoni 1990) können somit durch weitere Daten ergänzt und verglichen werden.

Das der vorliegenden Arbeit zugrundeliegende Korpus besteht aus den obigen Gründen aus Privatbriefen aus dem 19. Jahrhundert. Das 19. Jahrhundert bedeutet einen Umbruch in der Geschichte der deutschen Schriftsprache. In diesem Jahrhundert erlangten so viele Menschen Zugang zu den Fertigkeiten Lesen und Schreiben, wie nie zuvor. Im 19. Jahrhundert kamen zu diesen Fertigkeiten auch neue Schreibanlässe: Kriege, Auswanderung und Arbeitsmigration. Diese Gründe zwangen die damaligen Menschen zum Briefschreiben, denn früher war das (Brief-)schreiben keine alltägliche Routine, normalerweise war das Gespräch für die Kommunikation ein genügendes Mittel. (Elspaß 2007: 43) 
Die Auswandererbriefe dokumentieren die Sprache der unteren Schichten der Gesellschaft (Bauer, Kleinhändler, Arbeiter). Diese Leute haben nicht viel Zeit in der Schule verbracht, deshalb hatten sie einen einzigartigen Schreibstil: Sie schrieben fast so, als ob sie gesprochen hätten. Die Briefschreiber haben kaum Satzzeichen benutzt, ihre Texte enthalten phonetisch begründete Schreibvarianten (Elspaß 2005: 199 und 439).

In den Briefen wurden fünf Typen der Possessivität untersucht:

1.) Genitivkonstruktion (Haus des Vaters)

2.) Sächsischer Genitiv (Vaters Haus)

3.) Umschreibung mit von (Haus von dem Vater)

4.) Possessives Dativattribut (dem Vater sein Haus)

5.) Possessives Akkusativattribut (den Vater sein Haus).

Die Possessivpronomina, wie etwa mein, dein, ihr sowie die Konstruktionen, die zwar von enthalten, aber keine Possessivität ausdrücken (z.B. Komplemente in Form von Präpositionalphrasen wie zum Beispiel: einen Brief von meinen Freunden bekommen), wurden in der vorliegenden Arbeit nicht berücksichtigt. Des Weiteren sind auch adverbiale Verwendungen des Genitivs kein Gegenstand der Untersuchung.

Zur Variation der possessiven Genitiv- und Dativattribute sowie zu weiteren Formen der possessiven Attribute liegen bereits mehrere Untersuchungen vor. Elspaß (2005: 325-336) analysiert das possessive Dativattribut im 19. Jahrhundert und beschäftigt sich damit, wer, wann und in welchem Kontext zu welchen der beiden Ausdrucksformen (Genitiv- und possessives Dativattribut) neigte. Er weist nach, dass das possessive Dativattribut „auffällig häufig in der geschriebenen Alltagssprache unroutinierter Schreibender" $\mathrm{zu}$ finden ist (Elspaß 2005: 327-331), er führt jedoch keine quantitative Analyse der possessiven Attribute durch. Bei dem Erforschen der Texte 
„von der Umgangssprache näher stehenden“ (Elspaß 2005: 325) Autoren wie Raabe und Fontane (Admoni 1990) wurde festgestellt, dass der Anteil der Genitivkonstruktion bei diesen Texten nicht so hoch ist wie in den wissenschaftlichen oder Verwaltungstexten (Elspaß 2005: 325). Aber zur regionalen Verteilung gab es keine quantitative Untersuchung.

Ágel (2000: 1889) vertritt die Auffassung, dass ,in der nhd. Schriftsprache eher von einer Zunahme als von einem Rückgang des adnominalen Genitivs auszugehen ist“ (Elspaß 2005: 325). Von Polenz (1999: 345) behauptet, dass „der Genitiv als Attributkasus im amtlichen oder fachsprachlichen Stil >>relativ stabil geblieben ist $<<"$ (Elspaß, 2005: 326). Elspaß behauptet, dass der Gebrauch der analytischen Formen, besonders die von-Konstruktion, im 19. Jahrhundert in den Dialekten und den Umgangssprachen verstärkt wurde (2005: 326).

Die vorliegende Arbeit setzt sich deshalb zum Ziel, folgende Hypothesen mithilfe einer quantitativen Untersuchung zu überprüfen:

1.) Die Genitivkonstruktion überwiegt in den untersuchten Regionen.

2.) Der Anteil des possessiven Dativattributs erhöht sich im Laufe des 19. Jahrhunderts auf Kosten der Genitivkonstruktion.

3.) Der Anteil der von-Konstruktion erhöht sich im Laufe des 19. Jahrhunderts auf Kosten der Genitivkonstruktion.

4.) Der Anteil des possessiven Akkusativattributs ist nicht höher als $10 \%$.

5.) Bei der Verteilung der dominanten Varianten pro Region lassen sich großregionale Unterschiede erkennen. 
Diese Hypothesen werden an einem Korpus von Auswandererbriefen aus dem 19. Jahrhundert überprüft. Das Korpus wurde mir von Stephan Elspaß zur Verfügung gestellt, wofür ich mich an dieser Stelle bedanken möchte.

Zunächst werden die Terminologie und die Typen der possessiven Attribute beschrieben (Kapitel 2.1), dann wird der Forschungsstand zusammengefasst (Kapitel 2.2-2.5). Anschließend wird eine quantitative Untersuchung nach den obigen Aspekten durchgeführt (Kapitel 3.). Die Diskussion der Ergebnisse bzw. die Bestätigung/Verwerfung der obigen Hypothesen erfolgt im Kapitel 4.

\section{Theoretischer Hintergrund}

\subsection{Possessive Attribute im Deutschen}

Possessive Attribute, Possessorattribute können prototypischerweise eine Possessorrelation oder eine Zugehörigkeitsbeziehung ausdrücken (Zifonun 2017: 1568). Unter Possession ist die asymmetrische Beziehung zwischen einem Possessor und einem Possessum zu verstehen (Zifonun 2017: 1569). Im Deutschen nutzt man dafür zum Beispiel den attributiven Genitiv. Possessive Attribute im Deutschen können Possessorrelation ausdrücken: der Hut meines Vaters (der Vater hat einen Hut) drückt Possessorrelation aus, aber Tugend der Bescheidenheit ( ${ }^{*}$ die Bescheidenheit hat Tugend) nicht (Zifonun 2017: 1568).

Im Deutschen ist die Dependensmarkierung typisch, wie beim Ausdruck das Buch des Mädchens. Hier trägt das Dependens den Marker und ist rechts verzweigt (Zifonun, 2017: 1581). Das Deutsche operiert auch mit einer 'formalen' Präposition (von), die bei der analytischen Realisierung der possessi- 
ven Attribute verwendet wird: das Buch von dem Mädchen (Zifonun 2017: 1601). Des Weiteren ist im Deutschen die Possessorrelation oder eine Zugehörigkeitsbeziehung als pränominale und postnominale Attribute auszudrücken. In einer pränominalen Nominalphrase steht der Possessor (hier Peter) vor dem Kopf (hier Hund), in einem postnominalen Attribut steht der Kopf vor dem Possessor:

Pränominal: Peters Hund

Postnominal: Hund von Peter (Zifonun 2017: 1606).

Für den Gebrauch des pränominalen Genitivs gibt es im Deutschen besondere Beschränkungen (s. dazu Zifonun 2017: 1607).

\subsection{Tendenzen zur Verbreitung bzw. zum Rückgang der einzelnen Possessivattribute bis zum 19. Jahrhundert.}

Der verstärkte Gebrauch analytischer Formen, besonders des Präpositionalattributs, ist im 19. Jahrhundert in den Dialekten und den Umgangssprachen „bereits überwiegend eingetreten“ (Elspaß 2005: 326). „In den Dialekten wurde der adnominale Genitiv fast vollständig durch analytische Konstruktionen ersetzt“ (Elspaß 2005: 333). Trotz der Analytisierungstendenz kann man jedoch nicht von einem Rückgang des Genitivattributs im Deutschen ausgehen. Ágel (2000: 1889) behauptet, dass „in der nhd. Schriftsprache eher von einer Zunahme als von einem Rückgang des adnominalen Genitivs auszugehen ist“. Die Verteilung der einzelnen Varianten dürfte von den untersuchten Varietäten des Deutschen abhängen. Admoni (1990) stellte fest, dass „das Genitivattribut im 19. Jahrhundert eben- 
falls eine Tendenz zum häufigeren Gebrauch aufweise“ (vgl. Elspaß 2005: 325). Neben wissenschaftlichen und Verwaltungstexten untersuchte Admoni (1990) auch Romane, die von „der Umgangssprache näher stehenden“ (zit. n. Elspaß 2005: 325) Autoren wie Raabe und Fontane verfasst wurden und habe dabei „einen hohen Anteil von Präpositionalattributen“ festgestellt (Elspaß 2005: 325).

Deshalb ist es wichtig, die von unterschiedlich gebildeten Schreibern geschaffenen Quellen miteinander zu vergleichen. Die Verteilung der verschiedenen Varianten könnte auch vom Bildungsgrad der Textproduzenten abhängen. Es ist zu vermuten, dass der Anteil der Genitivkonstruktion in wissenschaftlichen, literarischen Texten und in der Verwaltungssprache höher ist als in den alltagssprachlichen Texten, wie zum Beispiel in den oben genannten Auswandererbriefen. Diese Briefe können ein differenzierteres Bild über den Gebrauch der possessiven Attribute im 19. Jahrhundert zeigen. Dadurch kann es auch deutlicher werden, wie sich der Gebrauch im Laufe des 19. Jahrhunderts änderte.

Die Tendenzen zur Verbreitung bzw. zum Rückgang der untersuchten Varianten des Possessivattributs werden von Elspaß (2005: 325-326) wie folgt zusammengefasst: „eine Verdoppelung der Präpositionalattribute in der Verwaltungssprache geht sicherlich mit kanzleisprachlichen Tendenzen zur Aufschwellung von Elementarsätzen und zur Nominalisierung einher“. Dabei bezieht er sich auf von Polenz (1999: 345), der feststellt, dass „der Genitiv als Attributkasus im amtlichen oder fachsprachlichen Stil $>>$ relativ stabil geblieben ist $<<$ “ (Elspaß 2005: 326).

Die Präpositionalattribute mit von (Haus von dem Vater) waren schon im 16. Jahrhundert in den Dialekten grammatika- 
lisiert und wurden sogar von Adelung „zur Vermeidung uneindeutiger Genitivkonstruktionen zugelassen“ (Elspaß 2005: 325). Das possessive Dativattribut (dem Vater sein Haus) als periphrastische Konstruktion gilt aus sprachhistorischem Aspekt als recht jung. Elspaß (2005) nimmt an, dass diese Konstruktion "sicher erst ab dem 15. Jahrhundert greifbar“ ist, „zunächst nur in niederdeutschen und oberdeutschen, auffälligerweise jedoch nicht in mitteldeutschen Quellen“ (Elspaß 2005: 326). Aber in der gesprochenen Sprache ist es seit langem vorhanden. Hingegen gehört diese Variante auch bis heute nicht zum Standard und ist ausgesprochen umgangssprachlich. Im Briefkorpus kommt diese Konstruktion oft vor, wobei es festzustellen ist, dass „für die Pronominalisierung des attribuierenden ersten Teils nur die 3. Person in Frage kommt“ (Elspaß 2005: 327). Weiterhin trifft die Bezeichnung Dativattribut „den Sachverhalt natürlich nicht ganz: Personennamen ohne Artikel oder Pronomen lassen keine Kasusmarkierung erkennen“ (Elspaß 2005: 331).

\subsection{Zur regionalen Verbreitung der einzelnen Possessivattribute}

Die regionale Verteilung der Varianten ist nicht eindeutig (Elspaß 2005: 332). Die Übersichtskarten (s. dazu Abb. 1 weiter unten) von Koß (1983) zeigen keinen Beleg für possessives Dativattribut in westfälischen, ostpommerschen und schlesischen Dialekten (Elspaß 2005: 332). Stattdessen ist auf den Karten der 'erhaltene Genitiv' markiert (Elspaß 2005: 332). Dem widerspricht, dass die Untersuchungen von Elspaß in den Briefen, die von Westfalen, Ostpommern und Schlesien stammenden Schreibern erzeugt wurden, Belege für possessive Da- 
tivattribute liefern (Elspaß 2005: 332). Dieses Phänomen lässt sich wie folgt erklären:

1.) In den westfälischen, ostpommerschen und schlesischen Dialekten existierte und existiert die Konstruktion, aber die Übersichtskarte ist unvollständig. (Koß stützte sich zur Anfertigung der Karten auf Sekundärquellen, u.a. auf die Belege von Behaghel 1923, s. dazu Elspaß 2005: 332).

2.) „Die Konstruktion ist nicht nur dialektal basiert.“ In diesem Fall kannten die Schreiber die Konstruktion aus einer großregionalen Umgangssprache (Elspaß 2005: 332).

Die Karten von Henn-Memmesheimer (1986) basieren auf Primärquellen und bestätigen Behaghels Beobachtungen, dass der Dativ in den 1920er Jahren „fast im ganzen Gebiet verbreitet" war. (Behaghel 1923: 638).

Das possessive Dativattribut wurde neben dem postnominalen präpositionalen Attribut mit von ,in der Alltagssprache des 19. Jahrhunderts voll grammatikalisiert“ (Elspaß 2005: 333). Zuerst wurden diese parallel zur alten flexivischen Konstruktion verwendet, dann haben sie immer mehr an Boden gewonnen und die synthetische Form weitgehend abgelöst. „In den Dialekten wurde der adnominale Genitiv fast vollständig durch analytische Konstruktionen ersetzt“ (Elspaß 2005: 333).

\subsection{Zur Funktionalität der einzelnen Possessivattribute}

Ursprünglich ersetzte die Präpositionalphrase mit von die für heute ausgestorbene ablativische Funktion. Das possessive Pronomen mit Dativ trat an die Stelle der possessiven Funktion, d.h., das possessive Pronomen mit Dativ ist als Ersatz für den Genitivus possessivus, der Besitzverhältnisse, und für den 
attributiven Genitiv, der ein Haben und/oder eine Zugehörigkeit ausdrückt, zu betrachten (Elspaß 2005: 333). Die analytischen Formen dienten und dienen dazu, die Zweifelsfälle eindeutiger auszudrücken (Elspaß 2005: 333). Ein solcher Zweifelsfall könnte die Konstruktion das Gemälde Tizians sein. Dabei wäre die Frage, ob Tizian der Besitzer oder der Schöpfer ist. Die Mehrdeutigkeit ist mit den folgenden beiden analytischen Formen aufzulösen (Beispiele nach Elspaß 2005: 333):

a) Das Gemälde von Tizian (Tizian als Schöpfer des Gegenstandes)

b) Dem Tizian sein Gemälde (Tizian als Besitzer des Gegenstandes)

Wie erwähnt, wurde die von-Konstruktion recht früh grammatikalisiert und neben seinem Gebrauch waren die possessiven Dativ- und Genitivattribute "deutlich weniger frequent“ (Elspaß 2005: 333). Marie-Elisabeth Fritzes (1976) Untersuchungen zufolge lag der Anteil der von-Konstruktion im Zeitraum 1470-1530 im Durchschnitt bei 19,7\%; demgegenüber ist diese Zahl in der fraglichen Periode für die possessiven Dativund Genitivattribute 0,1\% (Elspaß 2005: 333). Danach - zwischen 1670 und 1730 - ist eine Progression der possessiven Dativ- und Genitivattribute zu beobachten: Der Anteil der vonKonstruktion lag bei 9,9\%, während der Anteil der pränominalen Attribute auf 1,1\% gestiegen ist (Elspaß 2005: 334).

Die Untersuchungen von Elspaß (2005) beschäftigen sich mit drei Varianten, die Possessivität ausdrücken. In der vorliegenden Arbeit werden zwei weitere Varianten berücksichtigt, nämlich der sächsische Genitiv (Peters Haus) und das possessive Akkusativattribut (den Vater sein Haus). Das possessive Akkusativattribut wird nach Koß (1983: 1245) v.a. in den niederdeutschen Dialekten verwendet (vgl. Abb. 1): 


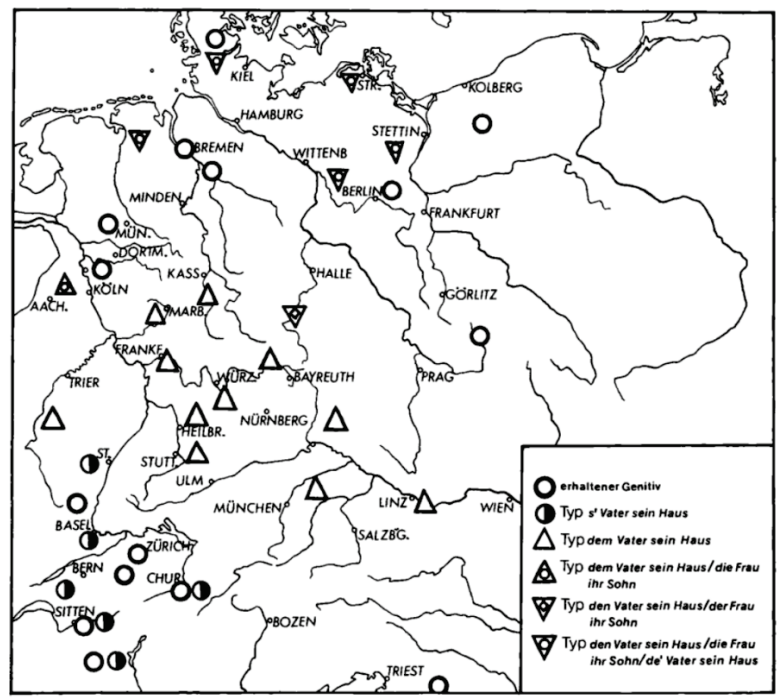

Abb. 1. Erhaltener Genitiv und Pronominalfügungen in den deutschen Dialekten nach Koß (1983: 1244)

Das possessive Akkusativattribut (den Vater sein Haus/die Frau ihr Sohn) erscheint nach Koß (1983: 1245) in einigen Dialekten nur bei Maskulina (Thüringisch), in anderen nur bei Feminina (Ripuarisch).

\subsection{Fazit}

Zur Possessivität und zur arealen Verbreitung der verschiedenen Formen liegen also mehrere Untersuchungen vor. Man kann auch die Geschichte einzelner Varianten in der historischen Linguistik nachverfolgen und daneben hat man über die Häufigkeit der einzelnen Varianten einige Informationen, bei zahlreichen Fällen sind die Ergebnisse auch mit Karten illustriert (Henn-Memmesheimer 1986; Koß 1983). Aber die Karten können nicht immer vollständig sein. Wie erwähnt erscheinen bei Koß (1983) in den westfälischen, ostpommerschen und 
schlesischen Dialekten keine Belege für das possessive Dativattribut (Elspaß 2005: 332). Die Übersichtskarten von HennMemmesheimer (1986) stammen aus den 80er Jahren, infolgedessen konnten die Belege sich nur auf das Gebiet der ehemaligen Bundesrepublik Deutschland beschränken.

Ein umfassendes Bild bietet die Karte von Elspaß und Möller (2003ff.) über den arealen Gebrauch des possessiven Attributs am Anfang des 21. Jahrhunderts (http://www.atlas-alltagssprache.de/attribut/):

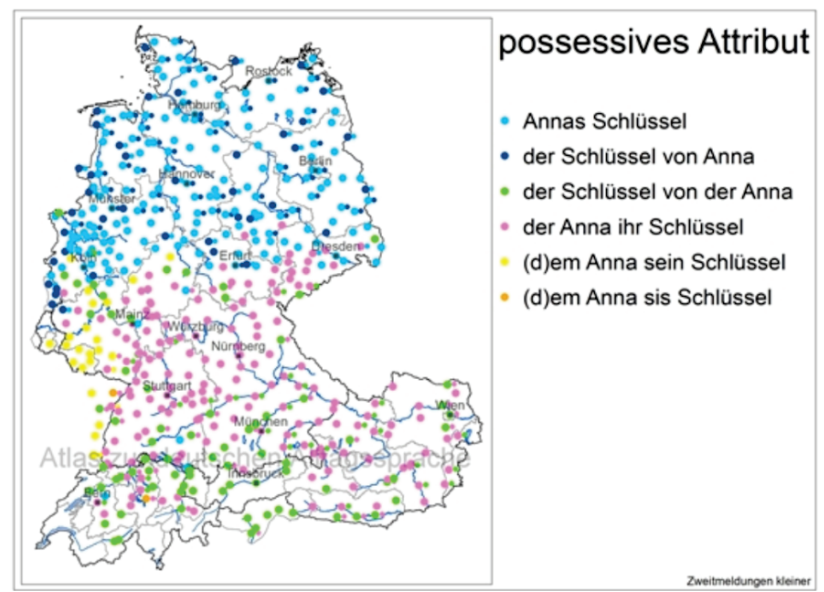

Abb. 2: Possessive Attribute in der Alltagssprache der Gegenwart

Die Daten zum Atlas zur deutschen Alltagssprache wurden mit Fragebogen erhoben, da die Forscher die gesprochene Umgangssprache und nicht die Standardsprache untersucht haben (http://www.atlas-alltagssprache.de/). Zunächst zeigt sich ein Nord-Süd-Unterschied: Es ist zu sehen, dass der sächsische Genitiv (Annas Schlüssel) und die Variante der Schlüssel von Anna in den norddeutschen Gebieten dominant sind, während die anderen Varianten im Süden wie folgt verteilt sind: Das possessive Dativattribut (der Anna ihr Schlüssel) ist eine typi- 
sche Variante in den mittel- und oberdeutschen Regionen. Die Variante der Schlüssel von der Anna ist fast ausschließlich auf Süddeutschland, Westösterreich und die deutschsprachige Schweiz beschränkt. Am westlichen Rande von Südwestdeutschland wird ebenso das possessive Dativattribut verwendet, aber mit einem anderen Genus (dem Anna sein Schlüssel). Die Variante (d)em Anna sis Schlüssel ist nur vereinzelt, in einigen Randgebieten zu finden. Die Variation der possessiven Attribute in der Alltagssprache der Gegenwart weist deutliche regionale Unterschiede auf. Aber es gibt keine Untersuchung in Bezug auf das 19. Jahrhundert, deshalb ist ein Vergleich mit den vergangenen Zuständen schwierig. Im Folgenden soll u.a. empirisch überprüft werden, ob die im Kapitel 1 formulierte Hypothese über regionale Unterschiede bei der Variation der possessiven Attribute in der Alltagssprache des 19. Jahrhunderts bestätigt werden kann.

\section{Analyse des Briefkorpus}

\subsection{Zum Korpus}

Stephan Elspaß (2005) hat in seiner Monografie das geschriebene Alltagsdeutsch im 19. Jahrhundert anhand eines Korpus aus Auswandererbriefen untersucht. Die verschiedenen Disziplinen verfügen heute über ca. 10000 überlieferte Auswandererbriefe aus dem 19. Jahrhundert. Elspaß (2005) hat davon ca. 650 analysiert (Elspaß 2005: 68), diese Briefe wurden mir zur Verfügung gestellt. Das Briefkorpus enthält nach Elspaß (2005: 68) ca. 375.000 Wortformen. Für die vorliegende Analyse wurden 465 Briefe mit einem Gesamtumfang von ca. 240000 Wortformen ausgewählt.

Die Briefe enthalten Metadaten, die sich auf die Schreibenden beziehen. Den Metadaten sind u.a. die Herkunft, die Religion, der 
Beruf und die Schulbildung der Schreibenden abzulesen. ist. Die Verfasser der Briefe sind Auswanderer oder die Verwandten, Freunde der ausgewanderten Personen, die meist aus den Regionen des heutigen Deutschlands stammten, aber manche Schreiber lebten - vor der Auswanderung - in französischen oder in belgischen Gebieten. Betrachtet man die Religion und die Konfession der Schreibenden, findet man unter denen Christen - Protestanten und Katholiken - und Juden. Was die Berufe der Schreibenden betrifft, sind sie oft Bauer, Arbeiter (Schuhmacher, Tischler, Schneider) und - im Fall einer Verfasserin - Hausfrauen. Daneben gibt es aber auch manche Kunstmaler und Dorflehrer. Die Alter der Briefschreiber sind auch unterschiedlich. Neben den persönlichen Daten sind auch die bibliografischen Informationen abzulesen, da auch die Quellen angegeben sind.

Zudem wurden die Texte regional eingeordnet. Diese regionale Einordnung und die Einordnung nach Zeitperioden wurden für die Bestimmung von Teilkorpora verwendet. Mein Korpus ist aber wegen der Quellenlage weder zeitlich noch regional ausgeglichen (vgl. Elspaß 2005: 68).

\begin{tabular}{|c|c|c|c|c|}
\hline $\begin{array}{c}\text { Zeitabschnitte } \\
\text { Regionen }\end{array}$ & $\begin{array}{c}1800- \\
1825\end{array}$ & $\begin{array}{c}1826- \\
1839\end{array}$ & $\begin{array}{c}1840- \\
1899\end{array}$ & insg. \\
\hline $\begin{array}{c}\text { Wod } \\
\text { (Westoberdeutsch) }\end{array}$ & 0 & 0 & 42273 & 42.273 \\
\hline $\begin{array}{c}\text { Nod } \\
\text { (Nordoberdeutsch) }\end{array}$ & 7613 & 2788 & 2526 & 12.927 \\
\hline $\begin{array}{c}\text { Ood } \\
\text { (Ostoberdeutsch) }\end{array}$ & 4889 & 1180 & 19.732 & 25.801 \\
\hline $\begin{array}{c}\text { Wmd } \\
\text { (Westmitteldeutsch) }\end{array}$ & 36.236 & 32.454 & 21.249 & 89.939 \\
\hline $\begin{array}{c}\text { Omd } \\
\text { (Ostmitteldeutsch) }\end{array}$ & 6258 & 5246 & 6441 & 17.945 \\
\hline $\begin{array}{c}\text { Wnd } \\
\text { (Westniederdeutsch) }\end{array}$ & 0 & 0 & 29.383 & 29.383 \\
\hline $\begin{array}{c}\text { Ond } \\
\text { (Ostniederdeutsch) }\end{array}$ & 0 & 0 & 21.386 & 21.386 \\
\hline ins. & 54.996 & 41.668 & 142.990 & 239.654 \\
\hline
\end{tabular}

Tabelle 1: Die Größe der Teilkorpora 


\subsection{Ergebnisse}

Im Folgenden sollen die Ergebnisse der quantitativen Analyse nach Zeitabschnitten gegliedert aufgeführt werden. Es ist wichtig zu betonen, dass diese Ergebnisse wegen der geringeren Belegzahl kein repräsentatives Bild über die Veränderungen der possessiven Attribute im 19. Jahrhundert geben.

\subsubsection{Analyse der Verteilung der Varianten im Zeitabschnitt zwischen 1800 und 1825}

Anhand der folgenden Tabelle ist zu sehen, wie die einzelnen possessiven Attribute zwischen 1800 und 1825 in den angegebenen Regionen verteilt waren.

\begin{tabular}{|c|c|c|c|c|c|c|c|c|}
\hline $\begin{array}{l}1800- \\
1825\end{array}$ & Wod & Nod & Ood & Wmd & Omd & Wnd & Ond & $\begin{array}{c}\text { Insge- } \\
\text { samt }\end{array}$ \\
\hline Genitiv & - & $\begin{array}{c}19 \\
(82,6 \%)\end{array}$ & $\begin{array}{c}1 \\
(8,33 \%)\end{array}$ & $\begin{array}{c}109 \\
(65,26 \%)\end{array}$ & $\begin{array}{c}1 \\
(50 \%)\end{array}$ & - & - & $\begin{array}{c}130 \\
(100 \%)\end{array}$ \\
\hline $\begin{array}{c}\text { Um- } \\
\text { schrei- } \\
\text { bung } \\
\text { mit von }\end{array}$ & - & - & $\begin{array}{c}4 \\
(33,33 \%)\end{array}$ & $\begin{array}{c}20 \\
(11 \\
97 \%)\end{array}$ & - & - & - & $\begin{array}{c}24 \\
(100 \%)\end{array}$ \\
\hline $\begin{array}{c}\text { Posses- } \\
\text { sives } \\
\text { Dati- } \\
\text { vattri- } \\
\text { but }\end{array}$ & - & $\begin{array}{c}1 \\
(4,34 \%)\end{array}$ & $\begin{array}{c}4 \\
(33,33 \%)\end{array}$ & $\begin{array}{c}15 \\
(8,98 \%)\end{array}$ & $\begin{array}{c}1 \\
(50 \%)\end{array}$ & - & - & $\begin{array}{c}21 \\
(100 \%)\end{array}$ \\
\hline $\begin{array}{c}\text { Posses- } \\
\text { sives } \\
\text { Akku- } \\
\text { sati- } \\
\text { vattri- } \\
\text { but } \\
\end{array}$ & - & $\begin{array}{c}3 \\
(13,04 \%)\end{array}$ & $\begin{array}{c}1 \\
(8,33 \%)\end{array}$ & $\begin{array}{c}8 \\
(4,79 \%)\end{array}$ & - & - & - & $\begin{array}{c}12 \\
(100 \%)\end{array}$ \\
\hline $\begin{array}{l}\text { Sächsi- } \\
\text { scher } \\
\text { Genitiv }\end{array}$ & - & - & $\begin{array}{c}2 \\
(16,66 \%)\end{array}$ & $\begin{array}{c}15 \\
(8,98 \%)\end{array}$ & - & - & - & $\begin{array}{c}17 \\
(100 \%)\end{array}$ \\
\hline $\begin{array}{c}\text { Beson- } \\
\text { derhei- } \\
\text { ten }\end{array}$ & - & - & - & - & - & - & - & - \\
\hline $\begin{array}{l}\text { Insge- } \\
\text { samt }\end{array}$ & 0 & $\begin{array}{c}23 \\
(100 \%)\end{array}$ & $\begin{array}{c}12 \\
(100 \%)\end{array}$ & $\begin{array}{c}167 \\
(100 \%)\end{array}$ & $\begin{array}{c}2 \\
(100 \%)\end{array}$ & 0 & 0 & $\begin{array}{c}204 \\
(100 \%)\end{array}$ \\
\hline
\end{tabular}

Tabelle 2: Die Verteilung der einzelnen possessiven Attribute zwischen 1800 und 1825 
Da das Teilkorpus keine Belege aus dem Wob, Wnd und Ond enthält und im Ostmitteldeutschen nur zwei auftauchen, werden diese Regionen in der quantitativen Analyse nicht berücksichtigt. Sowohl in der westmitteldeutschen als auch in der nordoberdeutschen Region liegt der Anteil der Genitivkonstruktion bei mehr als 50\%. Es ist aber interessant, dass diese Zahl in der ostoberdeutschen Region weniger als 9\% ist, während die von-Konstruktion und das possessive Dativattribut mit 33,33\% überwiegen. Dies stimmt mit Admonis (1990) Ergebnissen überein, der bei der Untersuchung der Romane von Fontane und Raabe „einen hohen Anteil von Präpositionalattributen“ festgestellt hat. (s. Kapitel 2.2; Elspaß 2005: 326).

\subsubsection{Analyse der Verteilung der Varianten zwischen 1800 und 1839}

Im Folgenden werden die Ergebnisse zum nächsten Zeitabschnitt des 19. Jahrhunderts diskutiert und die Daten in den Tabellen 2 und 3 miteinander verglichen. 


\begin{tabular}{|c|c|c|c|c|c|c|c|}
\hline $1826-1839$ & $\begin{array}{c}\text { Wod } \\
1826- \\
1839\end{array}$ & $\begin{array}{c}\text { Nod } \\
1826- \\
1839\end{array}$ & $\begin{array}{c}\text { Ood } \\
1826-\end{array}$ & $\begin{array}{c}\text { Wmd } \\
1826- \\
1839\end{array}$ & $\begin{array}{c}\text { Omd } \\
1826- \\
1839\end{array}$ & $\begin{array}{c}\text { Wnd } \\
1826- \\
1839\end{array}$ & $\begin{array}{c}\text { Ond } \\
1826- \\
1839\end{array}$ \\
\hline Genitiv & - & $\begin{array}{c}1 \\
(14,28 \%)\end{array}$ & - & $\begin{array}{c}78 \\
(60,46 \%)\end{array}$ & $\begin{array}{c}12 \\
(75 \%)\end{array}$ & - & - \\
\hline $\begin{array}{c}\text { Umschreibung } \\
\text { mit von }\end{array}$ & - & $\begin{array}{c}3 \\
(42,85 \%)\end{array}$ & - & $\begin{array}{c}17 \\
(13,17 \%)\end{array}$ & $\begin{array}{c}2 \\
(12,5 \%)\end{array}$ & - & - \\
\hline $\begin{array}{c}\text { Possessives } \\
\text { Dativattribut }\end{array}$ & - & $\begin{array}{c}2 \\
(28,57 \%)\end{array}$ & - & $\begin{array}{c}114 \\
(10,85 \%)\end{array}$ & - & - & - \\
\hline $\begin{array}{c}\text { Possessives } \\
\text { Akkusativattri- } \\
\text { but }\end{array}$ & - & - & - & $\begin{array}{c}4 \\
(3,1 \%)\end{array}$ & - & - & - \\
\hline 1826-1839 & $1826-$ & $\begin{array}{c}1826- \\
1839\end{array}$ & $\begin{array}{c}1826- \\
1839\end{array}$ & $\begin{array}{c}1826- \\
1839\end{array}$ & $\begin{array}{c}1826- \\
1839\end{array}$ & $\begin{array}{c}1826- \\
1839\end{array}$ & $\begin{array}{c}1826- \\
1839\end{array}$ \\
\hline $\begin{array}{c}\text { Sächsischer } \\
\text { Genitiv }\end{array}$ & - & $\begin{array}{c}1 \\
(14,28 \%)\end{array}$ & - & $\begin{array}{c}11 \\
(8,52 \%)\end{array}$ & $\begin{array}{c}2 \\
(12,5 \%)\end{array}$ & - & - \\
\hline $\begin{array}{c}\text { Besonderheiten } \\
\text { Insgesamt }\end{array}$ & - & - & - & $\begin{array}{c}5 \\
(3,87 \%)\end{array}$ & - & - & - \\
\hline
\end{tabular}

Tabelle 3: Die Verteilung der einzelnen possessiven Attribute zwischen 1826 und 1839

Auch in dem Zeitabschnitt 1826-39 mangelt es an Belegen aus einigen Regionen. In diesem Zeitabschnitt scheint die Genitivkonstruktion in der westmitteldeutschen Region immer noch zu überwiegen, aber ihr Anteil geht leicht zurück. Dasselbe ist auch bei dem sächsischen Genitiv zu beobachten. Der Anteil der Genitivkonstruktion wird auch in der nordoberdeutschen Region zurückgedrängt und noch dazu überwiegt die Umschreibung mit von mit mehr als $40 \%$. Damit wächst auch der 
Anteil des possessiven Dativattributs. In dieser Region erscheint auch das possessive Akkusativattribut. Wäre der Zeitabschnitt 1800-1825 aus dem Omd nicht außer Acht gelassen worden, könnte man feststellen, dass der Anteil der Genitivkonstruktion um 25\% gewachsen ist. Aber durch die Belegzahl der Tabelle 2 können diese Ergebnisse nicht bestätigt werden. In dieser Region überwiegt auch die Gentivkonstruktion mit einer hohen Prozentzahl (75\%) und daneben erscheinen nur die Von-Konstruktion und der sächsische Genitiv mit 12,5\%.

Die Belege der ostmitteldeutschen Region zwischen 1826 und 1839 reichen aus, um sie mit den Zahlen zwischen 1840 und $1899 \mathrm{zu}$ vergleichen.

\subsubsection{Analyse der Verteilung der Varianten zwischen 1840 und 1899}

Die Teilkorpus aus dem Zeitabschnitt zwischen 1840-1899 ist größer als die oben beschriebenen Teilkorpora und umfasst ca. 140.000 Wortformen.

Es ist eindeutig zu sehen, dass der sächsische Genitiv im Zeitabschnitt 1840-99 in allen untersuchten Regionen präsent ist, nicht wie in den ostmitteldeutschen und nordoberdeutschen Gebieten zwischen 1800 und 1825; und in der ostmitteldeutschen Region in dem Zeitabschnitt zwischen 18261839, wo es keine Belege für den sächsischen Genitiv gibt. Bei dem Vergleich der Zeitabschnitte 1826-39 und 1840-99 lässt sich die Konsequenz ziehen, dass die Genitivkonstruktion in dem letzten Drittel des Jahrhunderts zurückgedrängter ist; und nicht nur die Genitivkonstruktion, sondern auch die Umschreibung mit von. Ausnahmen sind die Anteile der vonKonstruktion in den ost- und westmitteldeutschen Gebieten, 
wo ihr Anteil sich erhöht. Demgegenüber nimmt der Anteil des possessiven Dativattributs zu. Der Anteil des sächsischen Genitivs erhöht sich nur in der ostmitteldeutschen Region, in westmitteldeutschen und in nordoberdeutschen Gebieten geht er zurück.

\begin{tabular}{|c|c|c|c|c|c|c|c|}
\hline $1840-1899$ & $\begin{array}{c}\text { Wod } \\
1840- \\
1899\end{array}$ & $\begin{array}{c}\text { Nod } \\
1840- \\
1899\end{array}$ & $\begin{array}{c}\text { Ood } \\
1840- \\
1899\end{array}$ & $\begin{array}{c}\text { Omd } \\
1840- \\
1899\end{array}$ & $\begin{array}{c}\text { Wmd } \\
1840- \\
1899\end{array}$ & $\begin{array}{c}\text { Wnd } \\
1840- \\
1899\end{array}$ & $\begin{array}{c}\text { Ond } \\
1840- \\
1899\end{array}$ \\
\hline Genitiv & $\begin{array}{c}84 \\
(61,76 \%)\end{array}$ & $\begin{array}{c}9 \\
(81,81 \%)\end{array}$ & $\begin{array}{c}36 \\
(73,46 \%)\end{array}$ & $\begin{array}{c}14 \\
(46,66 \%)\end{array}$ & $\begin{array}{c}21 \\
41,17 \%\end{array}$ & $\begin{array}{c}58 \\
(58 \%)\end{array}$ & $\begin{array}{c}81 \\
(71,68 \% \\
)\end{array}$ \\
\hline $\begin{array}{l}\text { Umschrei- } \\
\text { bung mit } \\
\text { von }\end{array}$ & $\begin{array}{c}29 \\
(21,32 \%)\end{array}$ & $\begin{array}{c}1 \\
(9,09 \%)\end{array}$ & $\begin{array}{c}3 \\
(6,12 \%)\end{array}$ & $\begin{array}{c}6 \\
(20 \%)\end{array}$ & $1019,6 \%$ & $\begin{array}{c}8 \\
(8 \%)\end{array}$ & $\begin{array}{c}17 \\
(15,04 \% \\
)\end{array}$ \\
\hline $\begin{array}{c}\text { Possessives } \\
\text { Dativ- } \\
\text { attribut }\end{array}$ & $\begin{array}{c}8 \\
(5,88 \%)\end{array}$ & - & $\begin{array}{c}5 \\
(10,2 \%)\end{array}$ & $\begin{array}{c}4 \\
(13,33 \%)\end{array}$ & $\begin{array}{c}15 \\
29,41 \%\end{array}$ & $\begin{array}{c}12 \\
(12 \%)\end{array}$ & $\begin{array}{c}5 \\
(4,42 \%)\end{array}$ \\
\hline $\begin{array}{c}\text { Possessives } \\
\text { Akkusativ- } \\
\text { attribut }\end{array}$ & - & - & $\begin{array}{c}1 \\
(2,04 \%)\end{array}$ & $\begin{array}{c}1 \\
(3,33 \%)\end{array}$ & $\begin{array}{c}1 \\
1,96 \%\end{array}$ & $\begin{array}{c}4 \\
(4 \%)\end{array}$ & - \\
\hline $\begin{array}{l}\text { Sächsi- } \\
\text { scher } \\
\text { Genitiv }\end{array}$ & $\begin{array}{c}15 \\
(11,02 \%)\end{array}$ & $\begin{array}{c}1 \\
(9,09 \%)\end{array}$ & $\begin{array}{c}4 \\
(8,16 \%)\end{array}$ & $\begin{array}{c}5 \\
(16,66 \%)\end{array}$ & $\begin{array}{c}4 \\
7,84 \%\end{array}$ & $\begin{array}{c}10 \\
(10 \%)\end{array}$ & $\begin{array}{c}10 \\
(8,84 \%)\end{array}$ \\
\hline $\begin{array}{l}\text { Besonder- } \\
\text { heiten }\end{array}$ & - & - & - & - & - & $\begin{array}{c}1 \\
(1 \%)\end{array}$ & - \\
\hline Insgesamt & $\begin{array}{c}136 \\
(100 \%)\end{array}$ & $\begin{array}{c}11 \\
(100 \%)\end{array}$ & $\begin{array}{c}49 \\
(100 \%)\end{array}$ & $\begin{array}{c}30 \\
(100 \%)\end{array}$ & $\begin{array}{c}51 \\
(100 \%)\end{array}$ & $\begin{array}{c}100 \\
(100 \%)\end{array}$ & $\begin{array}{c}113 \\
(100 \%)\end{array}$ \\
\hline
\end{tabular}

Tabelle 4: Die Verteilung der einzelnen possessiven Attribute zwischen 1826 und 1899

Es ist eindeutig zu sehen, dass der sächsische Genitiv im Zeitabschnitt 1840-99 in allen untersuchten Regionen präsent ist, nicht wie in den ostmitteldeutschen und nordoberdeutschen 
Gebieten zwischen 1800 und 1825; und in der ostmitteldeutschen Region in dem Zeitabschnitt zwischen 1826-1839, wo es keine Belege für den sächsischen Genitiv gibt. Bei dem Vergleich der Zeitabschnitte 1826-39 und 1840-99 lässt sich die Konsequenz ziehen, dass die Genitivkonstruktion in dem letzten Drittel des Jahrhunderts zurückgedrängter ist; und nicht nur die Genitivkonstruktion, sondern auch die Umschreibung mit von. Ausnahmen sind die Anteile der von-Konstruktion in den ost- und westmitteldeutschen Gebieten, wo ihr Anteil sich erhöht. Demgegenüber nimmt der Anteil des possessiven Dativattributs zu. Der Anteil des sächsischen Genitivs erhöht sich nur in der ostmitteldeutschen Region, in westmitteldeutschen und in nordoberdeutschen Gebieten geht er zurück.

In der nächsten Tabelle werden die Ergebnisse des Zeitabschnitts 1800-1826 und des Zeitabschnitts 1840-99 ausgewertet. 


\begin{tabular}{|c|c|c|c|c|c|c|c|c|c|c|c|}
\hline & $\begin{array}{c}\text { Wod } \\
1840- \\
1899 \\
\end{array}$ & $\begin{array}{c}\text { Nod } \\
1800- \\
1825 \\
\end{array}$ & $\begin{array}{c}\text { Nod } \\
1840- \\
1899\end{array}$ & $\begin{array}{c}\text { Ood } \\
1800- \\
1825\end{array}$ & $\begin{array}{c}\text { Ood } \\
1840- \\
1899\end{array}$ & $\begin{array}{c}\text { Omd } \\
1800- \\
1825\end{array}$ & $\begin{array}{c}\text { Omd } \\
1840- \\
1899\end{array}$ & $\begin{array}{c}\text { Wmd } \\
1800- \\
1825\end{array}$ & $\begin{array}{c}\text { Wmd } \\
1840- \\
1899\end{array}$ & $\begin{array}{c}\text { Wnd } \\
1840- \\
1899\end{array}$ & $\begin{array}{c}\text { Ond } \\
1840- \\
1899\end{array}$ \\
\hline Genitiv & $\begin{array}{c}84 \\
61,76 \%\end{array}$ & $\begin{array}{c}19 \\
82,6 \%\end{array}$ & $\begin{array}{c}9 \\
81,81 \%\end{array}$ & $\begin{array}{c}1 \\
8,33 \%\end{array}$ & $\begin{array}{c}36 \\
73,46 \%\end{array}$ & $\begin{array}{c}1 \\
50 \%\end{array}$ & $\begin{array}{c}14 \\
46,66 \%\end{array}$ & $\begin{array}{c}109 \\
65,26 \%\end{array}$ & $\begin{array}{c}21 \\
41,17 \%\end{array}$ & $\begin{array}{c}58 \\
58 \%\end{array}$ & $\begin{array}{c}81 \\
71,68 \%\end{array}$ \\
\hline $\begin{array}{l}\text { Umschreibung } \\
\text { mit von }\end{array}$ & $\begin{array}{c}29 \\
21,32 \%\end{array}$ & - & $\begin{array}{c}1 \\
9,09 \%\end{array}$ & $\begin{array}{c}4 \\
33,33 \%\end{array}$ & $\begin{array}{c}3 \\
6,12 \%\end{array}$ & - & $\begin{array}{c}6 \\
20 \%\end{array}$ & $\begin{array}{c}20 \\
11,97 \%\end{array}$ & $\begin{array}{c}10 \\
19,6 \%\end{array}$ & $88 \%$ & $\begin{array}{c}17 \\
15,04 \%\end{array}$ \\
\hline $\begin{array}{c}\text { Possessives } \\
\text { Dativattribut }\end{array}$ & $\begin{array}{c}8 \\
5,88 \%\end{array}$ & $\begin{array}{c}1 \\
4,34 \%\end{array}$ & - & $\begin{array}{c}4 \\
33,33 \%\end{array}$ & $\begin{array}{c}5 \\
10,2 \%\end{array}$ & $\begin{array}{c}1 \\
50 \%\end{array}$ & $\begin{array}{c}4 \\
13,33 \%\end{array}$ & $\begin{array}{c}15 \\
8,98 \%\end{array}$ & $\begin{array}{c}15 \\
29,41 \%\end{array}$ & $\begin{array}{c}12 \\
12 \%\end{array}$ & $\begin{array}{c}5 \\
4,42 \%\end{array}$ \\
\hline $\begin{array}{c}\text { Possessives } \\
\text { Akkusativattribut }\end{array}$ & - & $\begin{array}{c}3 \\
13,04 \%\end{array}$ & - & $\begin{array}{c}1 \\
8,33 \%\end{array}$ & $\begin{array}{c}1 \\
2,04 \%\end{array}$ & - & $\begin{array}{c}1 \\
3,33 \%\end{array}$ & $\begin{array}{c}8 \\
4,79 \%\end{array}$ & $\begin{array}{c}1 \\
1,96 \%\end{array}$ & $44 \%$ & - \\
\hline $\begin{array}{l}\text { Sächsischer } \\
\text { Genitiv }\end{array}$ & $\begin{array}{c}15 \\
11,02 \%\end{array}$ & - & $\begin{array}{c}1 \\
9,09 \%\end{array}$ & $\begin{array}{c}2 \\
16,66 \%\end{array}$ & $\begin{array}{c}4 \\
8,16 \%\end{array}$ & - & $\begin{array}{c}5 \\
16,66 \%\end{array}$ & $\begin{array}{c}15 \\
8,98 \%\end{array}$ & $\begin{array}{c}4 \\
7,84 \%\end{array}$ & $\begin{array}{c}10 \\
10 \%\end{array}$ & $\begin{array}{c}10 \\
8,84 \%\end{array}$ \\
\hline Besonderheiten & - & - & - & - & - & - & - & - & - & $\begin{array}{c}1 \\
1 \%\end{array}$ & - \\
\hline Insgesamt & $\begin{array}{c}136 \\
100 \%\end{array}$ & $\begin{array}{c}23 \\
100 \%\end{array}$ & $\begin{array}{c}11 \\
100 \%\end{array}$ & $\begin{array}{c}12 \\
100 \%\end{array}$ & $\begin{array}{c}49 \\
100 \%\end{array}$ & $\begin{array}{c}2 \\
100 \%\end{array}$ & $\begin{array}{c}30 \\
100 \%\end{array}$ & $\begin{array}{c}167 \\
100 \%\end{array}$ & $\begin{array}{c}51 \\
100 \%\end{array}$ & $\begin{array}{c}100 \\
100 \%\end{array}$ & $\begin{array}{c}113 \\
100 \%\end{array}$ \\
\hline
\end{tabular}

Tabelle 5: Die Verteilung der einzelnen possessiven Attribute zwischen 1800 und 1899 
Vergleicht man den ersten und den letzten Zeitabschnitt, lässt sich feststellen, dass in der westmitteldeutschen Region die Anzahl der Genitivkonstruktion bedeutend, um mehr als 20\% zurückgegangen ist. Dasselbe Schicksal zeigen das possessive Akkusativattribut und der sächsische Genitiv: beide sind nicht so stark wie die Genitivkonstruktion - zurückgedrängt.

Demgegenüber erhöht sich der Anteil der von-Konstruktion um fast $8 \%$. Noch spektakulärer ist die Veränderung der Prozentzahlen des possessiven Dativattributs: Die Progression ist nämlich mehr als 20\%. Die nordoberdeutsche Region zeigt an einigen Stellen andere Veränderungen. Auch hier ist der Anteil der Genitivkonstruktion zurückgedrängt, aber nur um 0,8\%. Die von-Konstruktion ist im Zeitabschnitt 1800-25 nicht belegt. Zwischen 1840 und 1899 ist ihr Anteil 9,09\%. Gegenüber der westmitteldeutschen Region gab es im Zeitabschnitt 1840-1899 keine Belege für das possessive Dativ- und für das possessive Akkusativattribut; aber in der ersten Zeitperiode waren ihre Anteile 4,34\% (possessives Dativattribut) und 13,08\% (possessives Akkusativattribut). Bei dem sächsischen Genitiv sieht die Verteilung der Belege genauso aus wie bei der von-Konstruktion: Kein Beleg in dem ersten Zeitabschnitt und 9,09\% in dem letzten. In dem ostoberdeutschen Gebiet gegenüber den nordoberdeutschen und westmitteldeutschen Regionen geht der Anteil der Genitivkonstruktion nicht zurück. Im Gegenteil: eine Erhöhung ist hier zu beobachten. Im Zeitabschnitt zwischen 1800 und 1825 liegt ihr Anteil an 8,33\%, während zwischen 1840 und 1899 an 73,46\%. Die von-Konstruktion nimmt auch nicht zu: von 33,33\% sinkt zu 6,12\%. Das geschieht auch mit dem possessiven Dativ- und possessiven Akkusativattributen. Der Anteil des possessiven Dativattributs liegt in der ersten Zeitperiode an 33,33\%, in der letzten ist diese 
Zahl nur 10,02\%. Der Anteil des possessiven Akkusativattributs zwischen 1800 und 1825 war ca. vier Mal so viel wie zwischen 1840 und 1899. Der Rücklauf bei dem sächsischen Genitiv ist nicht so groß: im Fall dieser Variante fällt der Anteil zur Hälfte zurück.

In den Tabellen 4 und 5 erscheinen auch die Belege und Prozentzahle der west- und ostniederdeutschen, beziehungsweise der west- und ostoberdeutschen Regionen. Bei jedem Gebiet überwiegt die Genitivkonstruktion und in zwei Fällen wird von der von-Konstruktion gefolgt. In dem westniederdeutschen Bereich werden das possessive Dativattribut und der sächsische Genitiv der Genitivkonstruktion folgen. In demselben Gebiet gab es einen Beleg für die Kategorie Besonderheiten, den ich als possessives Nominativattribut beschreiben würde: „der Peter sein Jungen Gaul“ (Brockmann 2, 15.10.67)).

Sowohl unter den aus den westoberdeutschen als auch in den aus den ostniederdeutschen Gebieten stammenden Briefen waren keine Belege für das possessive Akkusativattribut zu finden.

\section{Diskussion der Ergebnisse}

Im Folgenden werden die Ergebnisse zur arealen bzw. zur diachronen Verteilung der einzelnen Variante diskutiert. 


\begin{tabular}{|c|c|c|c|c|c|c|c|c|c|}
\hline $\begin{array}{c}\text { Region, Zeitab- } \\
\text { schnitte }\end{array}$ & $\begin{array}{c}\text { Wod } \\
1800- \\
1825\end{array}$ & $\begin{array}{c}\text { Wod } \\
1826- \\
1839\end{array}$ & $\begin{array}{c}\text { Wod } \\
1840- \\
1899\end{array}$ & $\begin{array}{c}\text { Ood } \\
1800- \\
1825\end{array}$ & $\begin{array}{c}\text { Ood } \\
1826- \\
1839\end{array}$ & $\begin{array}{c}\text { Ood } \\
1840- \\
1899\end{array}$ & $\begin{array}{c}\text { Nod } \\
1800- \\
1825\end{array}$ & $\begin{array}{c}\text { Nod } \\
1826- \\
1839\end{array}$ & $\begin{array}{c}\text { Nod } \\
1840- \\
1899\end{array}$ \\
\hline Genitiv & - & - & $\begin{array}{c}84 \\
(61,76 \%)\end{array}$ & $\begin{array}{c}1 \\
(8,33 \%)\end{array}$ & - & $\begin{array}{c}36 \\
(73,46 \%)\end{array}$ & $\begin{array}{c}19 \\
(82,6 \%)\end{array}$ & $\begin{array}{c}1 \\
(14,28 \%)\end{array}$ & $\begin{array}{c}9 \\
(81,81 \%)\end{array}$ \\
\hline $\begin{array}{c}\text { Umschreibung } \\
\text { mit von }\end{array}$ & - & - & $\begin{array}{c}29 \\
(10,32 \%)\end{array}$ & $\begin{array}{c}4 \\
(33,33 \%)\end{array}$ & - & $\begin{array}{c}3 \\
(6,12 \%)\end{array}$ & - & $\begin{array}{c}3 \\
(42,85 \%)\end{array}$ & $\begin{array}{c}1 \\
(9,09 \%)\end{array}$ \\
\hline $\begin{array}{c}\text { Possessives } \\
\text { Dativattribut }\end{array}$ & - & - & $\begin{array}{c}8 \\
(5,88 \%)\end{array}$ & $\begin{array}{c}4 \\
(33,33 \%)\end{array}$ & - & $\begin{array}{c}5 \\
(10,2 \%)\end{array}$ & $\begin{array}{c}1 \\
(4,34 \%)\end{array}$ & $\begin{array}{c}2 \\
(28,57 \%)\end{array}$ & - \\
\hline $\begin{array}{c}\text { Possessives } \\
\text { Akkusativattri- } \\
\text { but }\end{array}$ & - & - & - & 1 & - & 1 & 3 & - & - \\
\hline $\begin{array}{c}\text { Sächsischer } \\
\text { Genitiv }\end{array}$ & - & - & $\begin{array}{c}15 \\
(11,02 \%)\end{array}$ & $\begin{array}{c}2 \\
(16,66 \%)\end{array}$ & - & $\begin{array}{c}4 \\
(8,16 \%)\end{array}$ & - & $\begin{array}{c}1 \\
(14,28 \%)\end{array}$ & $(9,09 \%)$ \\
\hline $\begin{array}{c}\text { Besonderheiten } \\
\text { Insgesamt }\end{array}$ & - & - & - & - & - & - & - & - & - \\
\hline
\end{tabular}

Tabelle 6.1: Die Verteilung der possessiven Attribute im 19. Jahrhundert 


\begin{tabular}{|c|c|c|c|c|c|c|}
\hline $\begin{array}{c}\text { Zeitperiode, } \\
\text { Region }\end{array}$ & $\begin{array}{c}\text { Wmd } \\
1800-1825\end{array}$ & $\begin{array}{c}\text { Wmd } \\
1826-1839\end{array}$ & $\begin{array}{c}\text { Wmd } \\
1840-1899\end{array}$ & $\begin{array}{c}\text { Omd } \\
1800-1825\end{array}$ & $\begin{array}{c}\text { Omd } \\
1826-1839\end{array}$ & $\begin{array}{c}\text { Omd } \\
1840-1899\end{array}$ \\
\hline Genitiv & $109(65,26 \%)$ & $\begin{array}{c}78 \\
(60,46 \%)\end{array}$ & $\begin{array}{c}21 \\
(41,17 \%)\end{array}$ & $\begin{array}{c}1 \\
(50 \%)\end{array}$ & $\begin{array}{c}12 \\
(75 \%)\end{array}$ & $14(46,66 \%)$ \\
\hline $\begin{array}{c}\text { Umschreibung } \\
\text { mit von }\end{array}$ & $\begin{array}{c}20 \\
(11,97 \%)\end{array}$ & $\begin{array}{c}17 \\
(13,17 \%)\end{array}$ & $\begin{array}{c}10 \\
(19,6 \%)\end{array}$ & - & 2 & 6 \\
\hline $\begin{array}{c}\text { Possessives } \\
\text { Dativattribut }\end{array}$ & $\begin{array}{c}15 \\
(8,98 \%)\end{array}$ & $\begin{array}{c}14 \\
(10,85 \%)\end{array}$ & $\begin{array}{c}15 \\
(29,41 \%)\end{array}$ & $\begin{array}{c}1 \\
(50 \%)\end{array}$ & - & $(20 \%)$ \\
\hline $\begin{array}{c}\text { Possessives } \\
\text { Akkusativattribut }\end{array}$ & $\begin{array}{c}(4,79 \%) \\
(3,1 \%)\end{array}$ & $\begin{array}{c}1 \\
(1,96 \%)\end{array}$ & - & - & $\begin{array}{c}1 \\
(3,33 \%)\end{array}$ \\
\hline & - & $\begin{array}{c}5 \\
(3,87 \%)\end{array}$ & - & - & - & - \\
\hline Besonderheiten & 129 & 51 & 2 & 16 & 30 \\
\hline Insgesamt & $(100 \%)$ & $(100 \%)$ & $(100 \%)$ & $(100 \%)$ & $(100 \%)$ & $(100 \%)$ \\
\hline
\end{tabular}

Tabelle 6.2: Die Verteilung der possessiven Attribute im 19. Jahrhundert 


\begin{tabular}{|c|c|c|c|c|c|c|}
\hline $\begin{array}{l}\text { Zeitperiode, } \\
\text { Region }\end{array}$ & $\begin{array}{c}\text { Wnd } \\
1800-1825\end{array}$ & $\begin{array}{c}\text { Wnd } \\
1826-1839\end{array}$ & $\begin{array}{c}\text { Wnd } \\
1840-1899\end{array}$ & $\begin{array}{c}\text { Ond } \\
1800-1825\end{array}$ & $\begin{array}{c}\text { Ond } \\
1825-1839\end{array}$ & $\begin{array}{c}\text { Ond } \\
1840-1899\end{array}$ \\
\hline Genitiv & - & - & $\begin{array}{c}58 \\
(58 \%)\end{array}$ & - & - & $\begin{array}{c}81 \\
(71,68 \%)\end{array}$ \\
\hline $\begin{array}{l}\text { Umschreibung } \\
\text { mit von }\end{array}$ & - & - & $\begin{array}{c}8 \\
(8 \%)\end{array}$ & - & - & $\begin{array}{c}17 \\
(15,04 \%)\end{array}$ \\
\hline $\begin{array}{l}\text { Possessives } \\
\text { Dativattribut }\end{array}$ & - & - & $\begin{array}{c}12 \\
(12 \%)\end{array}$ & - & - & $\begin{array}{c}5 \\
(4,42 \%)\end{array}$ \\
\hline $\begin{array}{l}\text { Sächsischer } \\
\text { Genitiv }\end{array}$ & - & - & $\begin{array}{c}10 \\
(10 \%)\end{array}$ & - & - & $\begin{array}{c}10 \\
(8,84 \%)\end{array}$ \\
\hline Besonderheiten & - & - & $\begin{array}{c}1 \\
(1 \%)\end{array}$ & - & - & - \\
\hline Insgesamt & 0 & 0 & $\begin{array}{c}100 \\
(100 \%)\end{array}$ & 0 & 0 & $\begin{array}{c}113 \\
(100 \%)\end{array}$ \\
\hline
\end{tabular}

Tabelle 6.3: Die Verteilung der possessiven Attribute im 19. Jahrhundert 
Da im Fall der westoberdeutschen, westniederdeutschen und ostniederdeutschen Regionen nur Belege aus dem dritten Zeitabschnitt vorliegen, kann man hier die Veränderungen im Laufe des Jahrhunderts kaum bewerten. Diese Tabelle zeigt in Bezug auf die Genitivkonstruktion in den Ood- und NodRegionen keine eindeutige Tendenz. In den ostoberdeutschen Gebieten können nur zwei Zeitabschnitte in Betracht genommen werden und diese zeigen eine enorme Progression. Die nordoberdeutschen Regionen zeigen einen großen Rückgang, wenn man den ersten mit dem zweiten Zeitabschnitt vergleicht. Dies ist der Fall auch dann, wenn der erste und der dritte Zeitabschnitt verglichen werden, aber die Genitivkonstruktion ist hier nicht in so stark zurückgedrängt. Zwischen den Zeitperioden 1826-1839 und 1840-1899 sieht man aber eine Erhöhung des Anteils der Genitivkonstruktion in der nordoberdeutschen Region.

Der Anteil der Genitivkonstruktion geht in den mitteldeutschen Regionen zurück. Dieser Rückgang ist im Westmitteldeutschen eindeutig zu sehen. Wie erwähnt, liegt für den Zeitabschnitt 1800-1825 nur eine geringe Belegzahl aus dem Omd vor. Die von-Konstruktion zeigt die Veränderungen eindeutiger. Es gibt Zeitabschnitte, wo sich dafür kein Beleg befindet. Es lässt sich feststellen, dass der Anteil der von-Konstruktion in den mitteldeutschen Gebieten kontinuierlich zunimmt, während in den oberdeutschen Bereichen ihre Prozentzahl sinkt.

Eine Aussage über den possessiven Dativ zu formulieren ist problematisch. Anhand der Tabelle lässt sich keine eindeutige und einheitliche Tendenz feststellen. In Bezug auf die oberdeutschen Regionen könnte festgestellt werden, dass sich der Anteil des possessiven Dativattributs in der nordoberdeutschen Region erhöht, in den ostoberdeutschen Gebieten aber 
zurückgeht. Aber in der oberdeutschen Region fehlt in zwei Zeitabschnitten das possessive Dativattribut, deshalb ist es nicht empfehlenswert, solche Aussagen zu formulieren. Im mitteldeutschen Teilkorpus erscheint die Genitivkonstruktion am häufigsten. Die von-Konstruktion und das possessive Dativattribut rivalisieren für die zweite Stelle, aber in den meisten Fällen steht das possessive Dativattribut an der dritten Stelle. Ausnahme ist der Zeitabschnitt 1840-1899 im Wmd.

Über das possessive Akkusativattribut kann wegen der geringen Belegzahl nur in Bezug auf die westmitteldeutsche Region geäußert werden. Hier ist ein eindeutiger Rückgang zu sehen. Der sächsische Genitiv ist relativ stabil geblieben: sein Anteil ist in keinem Fall weniger als 7\% und nicht mehr als 17\%.

Die folgende Tabelle fasst die Daten aus dem Jahrhundert zusammen: 


\begin{tabular}{|c|c|c|c|c|c|c|c|c|}
\hline Regionen & Wod & Nod & Ood & Wmd & Omd & Wnd & Ond & Insgesamt \\
\hline Genitiv & $\begin{array}{c}84 \\
(61,76 \%)\end{array}$ & $\begin{array}{c}29 \\
(70,73 \%)\end{array}$ & $\begin{array}{c}37 \\
(60,65 \%)\end{array}$ & $\begin{array}{c}208 \\
(59,94 \%)\end{array}$ & $\begin{array}{c}27 \\
(56,25 \%)\end{array}$ & $\begin{array}{c}58 \\
(58 \%)\end{array}$ & $\begin{array}{c}81 \\
(71,68 \%)\end{array}$ & $\begin{array}{c}524 \\
(100 \%)\end{array}$ \\
\hline $\begin{array}{c}\text { Umschreibung } \\
\text { mit von }\end{array}$ & $\begin{array}{c}29 \\
(21,32 \%)\end{array}$ & $\begin{array}{c}4 \\
(9,75 \%)\end{array}$ & $\begin{array}{c}7 \\
(11,47 \%)\end{array}$ & $\begin{array}{c}47 \\
(13,54 \%)\end{array}$ & $\begin{array}{c}8 \\
(16,66 \%)\end{array}$ & $\begin{array}{c}8 \\
(8 \%)\end{array}$ & $\begin{array}{c}17 \\
(15,04 \%)\end{array}$ & $\begin{array}{c}120 \\
(100 \%)\end{array}$ \\
\hline $\begin{array}{c}\text { Possessives } \\
\text { Dativattribut }\end{array}$ & $\begin{array}{c}8 \\
(5,88 \%)\end{array}$ & $\begin{array}{c}3 \\
(7,31 \%)\end{array}$ & $\begin{array}{c}9 \\
(14,75 \%)\end{array}$ & $\begin{array}{c}44 \\
(12,68 \%)\end{array}$ & $\begin{array}{c}5 \\
(10,41 \%)\end{array}$ & $\begin{array}{c}12 \\
(12 \%)\end{array}$ & $\begin{array}{c}5 \\
(4,42 \%)\end{array}$ & $\begin{array}{c}86 \\
(100 \%)\end{array}$ \\
\hline $\begin{array}{c}\text { Possessives } \\
\text { Akkusativattribut }\end{array}$ & - & $\begin{array}{c}2 \\
(7,31 \%)\end{array}$ & $\begin{array}{c}2,27 \%) \\
(3,74 \%)\end{array}$ & $\begin{array}{c}1 \\
(2,08 \%)\end{array}$ & $\begin{array}{c}4 \\
(4 \%)\end{array}$ & - & $\begin{array}{c}23 \\
(100 \%)\end{array}$ \\
\hline Sächsischer Genitiv & $\begin{array}{c}15 \\
11,02 \%)\end{array}$ & $\begin{array}{c}2 \\
(4,87 \%)\end{array}$ & $\begin{array}{c}6 \\
(9,83 \%)\end{array}$ & $\begin{array}{c}30 \\
(8,64 \%)\end{array}$ & $\begin{array}{c}7 \\
(14,58 \%)\end{array}$ & $\begin{array}{c}10 \\
(10 \%)\end{array}$ & $\begin{array}{c}10 \\
(8,84 \%)\end{array}$ & $\begin{array}{c}80 \\
(100 \%)\end{array}$ \\
\hline Besonderheiten & - & - & - & $\begin{array}{c}5 \\
(1,44 \%)\end{array}$ & - & $\begin{array}{c}1 \\
(1 \%)\end{array}$ & - & 6 \\
$(100 \%)$ \\
\hline Insgesamt
\end{tabular}

Tabelle 7: Die Verteilung der possessiven Attribute im 19. Jahrhundert 
Aufgrund der obigen Daten lassen sich die in der Einleitung formulierten Hypothesen (vgl. Kapitel 1) wie folgt bewerten:

1.) Die Genitivkonstruktion überwiegt in den untersuchten Regionen.

Betrachtet man die Tabellen 6.1., 6.2., 6.3., sieht man, dass die Genitivkonstruktion nicht in jeder Region überwiegt und geht auch nicht kontinuierlich bzw. nicht in jedem Fall zurück. In den meisten Fällen überwiegt tatsächlich die Genitivkonstruktion, aber in der nordoberdeutschen Region zwischen 1826 und 1839 steht die von-Konstruktion an der ersten Stelle und wird nicht von der Genitivkonstruktion, sondern von dem possessiven Dativattribut gefolgt. Auch in dem ostoberdeutschen Gebiet verliert die Genitivkonstruktion gegenüber der vonKonstruktion und dem possessiven Dativattribut (ihre Anteile sind 33,33\%). Aber anhand der Tabelle 6 kann man sagen, dass in dem ganzen Jahrhundert die Genitivkonstruktion dominiert: ihr Anteil ist in jedem Fall mehr als 50\%, während der Anteil der anderen Typen nur einmal die $20 \%$ erreichen.

2.) Der Anteil des possessiven Dativattributs erhöht sich im Laufe des 19. Jahrhunderts auf Kosten der Genitivkonstruktion.

Die Hypothese kann nur durch die Daten zur westmitteldeutschen Region in den Tabellen 6.1, 6.2, 6.3 bestätigt werden. In derselben Tabelle sind die ostmitteldeutschen, die nordoberdeutschen und die ostoberdeutschen Datensätze unvollständig, deshalb zeigen sie keine eindeutigen Ergebnisse. Betrachtet man aber nur den ersten und den letzten Zeitabschnitt in dem ostmitteldeutschen Gebiet, ist die Information abzulesen, dass der Anteil des possessiven Dativattributs in der ostmitteldeutschen Region zurückgeht, während der Anteil der Genitivkon- 
struktion sich erhöht. Die Hypothese kann anhand der zur Verfügung stehenden, lückenhaften Daten nicht eindeutig bestätigt oder verworfen werden. Die Daten aus der nordoberdeutschen Region scheinen sie zwar zu bestätigen, das ist aber im Falle der ostoberdeutschen Region genau umgekehrt.

3.) Der Anteil der von-Konstruktion erhöht sich im Laufe des 19. Jahrhunderts auf Kosten der Genitivkonstruktion.

Im Fall der von-Konstruktion lässt sich dasselbe Phänomen wie beim possessiven Dativattribut beobachten: Nur die Datengrundlage zum Westmitteldeutschen ist ausreichend, und diese bestätigt die Hypothese. Berücksichtigt man auch die Regionen mit z.T. fehlenden Belegen, könnte das Ergebnis auch umgekehrt interpretiert werden: Nimmt der Anteil der Genitivkonstruktion $\mathrm{zu}$, geht die Prozentzahl der von-Konstruktion zurück.

4.) Der Anteil des possessiven Akkusativattributs ist nicht höher als $10 \%$.

Die vorletzte Vermutung kann nicht eindeutig bestätigt werden. In der nordoberdeutschen Region ist der Anteil des possessiven Akkusativattributs im Zeitabschnitt 1800-1825 mehr als $13 \%$. In der nordoberdeutschen Region, in der sich insgesamt nur wenige Belege finden, beträgt der Anteil des possessiven Akkusativattributs im 19. Jahrhundert insgesamt nur $7,31 \%$. Es soll jedoch bemerkt werden, dass dieser Wert in den anderen Regionen in keinem Fall überschritten wird.

5.) Bei der Verteilung der dominanten Varianten pro Region lassen sich großregionale Unterschiede erkennen. 
Anhand den Tabellen 6.1, 6.2, 6.3 ist diese Hypothese bestätigt, da die Genitivkonstruktion nicht in jeder Region und nicht in jedem Zeitabschnitt dominiert. Auch die von-Konstruktion und das possessive Dativattribut bekamen eine führende Rolle. Untersucht man die Daten des ganzen Jahrhunderts zusammen (Tabelle 7), kann diese Vermutung verworfen werden, weil, wie erwähnt, der Anteil der Genitivkonstruktion in jedem Fall mehr als 50\% ist. Diese bestätigt auch die folgende Sprachkarte, mit der auch die Ergebnisse von Elspaß und Möller (2003ff.) zu vergleichen sind. Die Karte habe ich aufgrund einer Abbildung in einem Beitrag aus der Deutschen Welle (Wasserrab o.J.) erstellt.

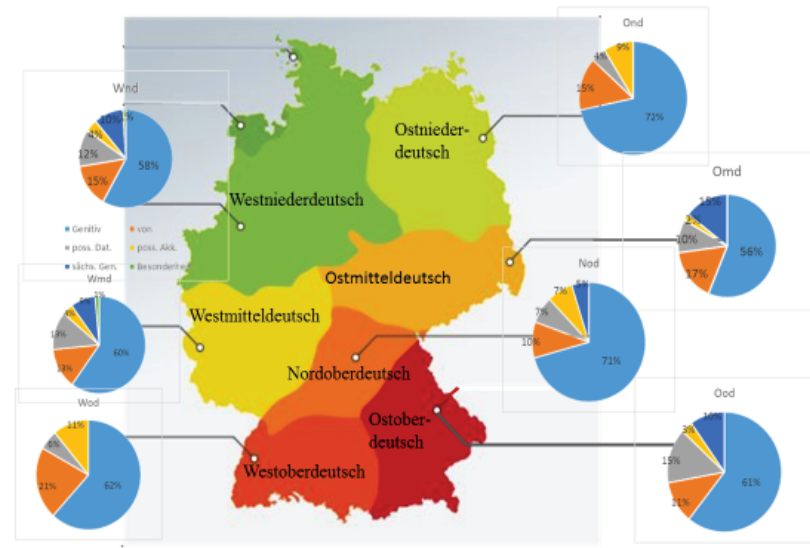

Abb. 3: Die Verteilung der possessiven Attribute im 19. Jahrhundert

Der Karte von Elspaß und Möller (2003ff.) lässt sich ein NordSüd-Unterschied ablesen. Demgegenüber sieht man diesen Unterschied in den Daten aus dem 19. Jahrhundert nicht. Die Verteilung der possessiven Attribute im 19. Jahrhundert war anhand der oberen Karte in allen Gebieten ähnlich. Die Genitivkonstruktion überwiegt, an der zweiten Stelle steht die vonKonstruktion. Ein Unterschied ist in der Reihenfolge der anderen possessiven Attribute zu finden. Zum Beispiel folgt der 
von-Konstruktion in den meisten Gebieten das possessive Dativattribut, eine Ausnahme ist das ostmitteldeutsche Bereich, wo der sächsische Genitiv mit $15 \%$ an der dritten Stelle steht. Des Weiteren ist der Anteil des possessiven Akkusativattributs in der nordoberdeutschen Region deutlich höher als in anderen Regionen.

\section{Zusammenfassung und Ausblick}

In der vorliegenden Arbeit wurde das Ziel gesetzt, die Verteilung der possessiven Attribute in der deutschen Alltagssprache im 19. Jahrhundert zu untersuchen und über den hochdeutschen Gebieten ein quantitatives Bild zu bekommen. Hinsichtlich der beschränkten Größe des zur Verfügung gestellten Korpus, können die Ergebnisse nur einen Einblick bieten und kein umfassendes Bild. Die bisherigen Forschungen konzentrierten sich zuerst auf die standarddeutschen Quellen, die mit den Texten „von der Umgangssprache näher stehenden“ (Elspaß 2005: 325) Autoren wie Raabe und Fontane (Admoni 1990) ergänzt wurden. Dabei wurde festgestellt, dass der Anteil der Genitivkonstruktion bei diesen Texten nicht so hoch ist wie in den wissenschaftlichen oder Verwaltungstexten (Elspaß 2005: 325). Aber, wie erwähnt, zur regionalen Verteilung gab es nur wenige quantitative Untersuchung. Anhand dieser Untersuchungen (vgl. Kapitel 1 und 2) habe ich die folgenden Hypothesen formuliert:

1.) Die Genitivkonstruktion überwiegt in den untersuchten Regionen.

Die Ergebnisse in Kapitel 4. zeigten, dass die Genitivkonstruktion nicht in allen Zeitabschnitten überwiegend war. Wie erwähnt, in der ostoberdeutschen Region zwischen 1800 und 
1825 überwieten die von-Konstruktion und das possessive Dativattribut mit 33,33\%; in dem nordoberdeutschen Gebiet zwischen 1826 und 1839 hatte die von-Konstruktion den größten Anteil. In dem ganzen Jahrhundert aber dominiert der Genitiv (Tabelle 6).

Da Elspaß (2005: 326) meint, dass der Gebrauch des Präpositionalattributs im Laufe des Jahrhunderts verstärkt wurde, konnten für die vorliegende Arbeit folgende Hypothesen formuliert werden:

2.) Der Anteil des possessiven Dativattributs erhöht sich im Laufe des 19. Jahrhunderts auf Kosten der Genitivkonstruktion.

3.) Der Anteil der von-Konstruktion erhöht sich im Laufe des 19. Jahrhunderts auf Kosten der Genitivkonstruktion.

Diese zwei Hypothesen konnten wegen der Quellenlage vor allem durch die Daten aus der westmitteldeutschen Region bestätigt werden. Dabei wurde der folgende Zusammenhang festgestellt: Nimmt der Anteil der Genitivkonstruktionen zu, gehen die Prozentzahlen der von-Konstruktion und des possessiven Dativattributs zurück.

Nach den Untersuchungen von Koß (1983) wird das possessive Akkusativattribut v.a. in niederdeutschen Dialekten, bei Maskulina aber auch in mitteldeutschen und teilweise bairischösterreichischen Gebieten verwendet, deshalb habe ich Folgendes vermutet:

4.) Der Anteil des possessiven Akkusativattributs ist nicht höher als $10 \%$.

Bis auf die nordoberdeutsche Region war der Anteil des possessiven Akkusativattributs unter 7\%. Der Gebrauch des possessi- 
ven Akkusativattributs konnte anhand des Korpus in den westoberdeutschen und ostniederdeutschen Regionen nicht nachgewiesen werden.

In Abb. 1 (s. weiter oben) lässt sich eine großregionale Verteilung der Possessivattribute in der Gegenwartssprache erkennen. Anhand dieser Karte habe ich die letzte Hypothese in Bezug auf das 19. Jahrhundert gestellt:

5.) Bei der Verteilung der dominanten Varianten der Possessivattribute pro Region lassen sich großregionale Unterschiede erkennen.

In einigen Regionen und in einigen Zeitabschnitten ist zwar die Genitivkonstruktion nicht dominant. Demgegenüber zeigt die Tabelle 7 eindeutig, dass in dem ganzen Jahrhundert der Genitiv überwiegt, deshalb lässt sich kein großregionaler Unterschied erkennen.

Aus den Daten lässt sich die Konsequenz ableiten, dass die Genitivkonstruktion überwiegt, ihr Anteil liegt an mehr als $50 \%$. In der nordoberdeutschen Region steht die von-Konstruktion an der ersten Stelle, in dem ostoberdeutschen Gebiet teilt sich die führende Rolle zwischen dem possessiven Dativattribut und der von-Konstruktion.

Es ist interessant, dass in den Spalten der Tabellen 6.1, 6.2, 6.3, wo der Anteil der Genitivkonstruktion weniger als 50\% ist, der Anteil des sächsischen Genitivs bei mehr als 14\% liegt. Diese sind in den Spalten Ood. 1800-1825, Nod. 1826-1839, Omd. 1840-1899 zu beobachten. Dazu kommt noch, dass der Anteil der Genitivkonstruktion mehr als $80 \%$ ist, wo der Anteil des sächsischen Genitivs bei weniger als $10 \%$ liegt oder wo es dafür keinen Beleg gibt. Das possessive Akkusativattribut scheint selten vorzukommen. Wenn es überhaupt belegt ist, 
findet es sich in den meisten Teilkorpora mit einem Anteil unter 5\%. Sein höchster Anteil ist 13,04\% im Zeitabschnitt 18001825 aus dem Nod.

In der vorliegenden Arbeit wurden die possessiven Attribute in Deutschland untersucht. In weiteren Untersuchungen könnten auch Briefe aus Österreich und der deutschsprachigen Schweiz analysiert werden, sofern die Quellenlage dies erlaubt. Da die Briefe auch Metadaten enthalten (vgl. Kapitel 3), könnte die Verteilung der possessiven Attribute zum Beispiel auch nach der Konfession der einzelnen Verfasser untersucht werden.

\section{Literaturverzeichnis}

AdA = Elspaß, Stephan / Robert Möller (2003ff.): Atlas zur deutschen Alltagssprache (AdA). Open-Access-Publikation: $<$ www.atlas-alltagssprache.de> (zuletzt gesehen: 21.6. 2021)

Admoni, Wladimir (1990): Historische Syntax des Deutschen. Tübingen: Niemeyer.

Ágel, Vilmos (2000): Syntax des Neuhochdeutschen bis zur Mitte des 20. Jahrhunderts. In: Besch, Werner et al. (Hrsg.): Sprachgeschichte. Ein Handbuch zur Geschichte der deutschen Sprache und ihrer Erforschung. Bd. 2. 2. Aufl. Berlin/New York: de Gruyter (HSK 2.2), 1855-1903.

Behaghel, Otto (1923): Deutsche Syntax. Eine geschichtliche Darstellung. Bd. I. Heidelberg: Winter.

Elspaß, Stephan (2005): Sprachgeschichte von unten: Untersuchungen zum geschriebenen Alltagsdeutsch im 19. Jahrhundert. Tübingen: Niemeyer (Reihe Germanistische Linguistik 263). 
Elspaß, Stephan (2007): Briefe und Tagebücher des 19. und 20. Jahrhundert als Quellen der Alltags- und Alltagssprachgeschichte. In: Ders. (Hrsg.): Neue Sprachgeschichte(n) (Themenheft der Zeitschrift Der Deutschunterricht, 59.3), 42-52.

Fritze, Marie-Elisabeth (1976): Bezeichnungen für den Zugehörigkeits- und Herkunftsbereich beim substantivischen Attribut. In: Kettmann, Gerhard/Schild, Joachim (Hrsg.): Zur Ausbildung der Norm der deutschen Literatursprache auf der syntaktischen Ebene (1470-1730). I. Der Einfachsatz. Berlin: Akademie-Verlag (Bausteine zur Sprachgeschichte des Neuhochdeutschen, 56/1), 417-476.

Henn-Memmesheimer, Beate (1986): Nonstandardmuster: ihre Beschreibung in der Syntax und das problem ihrer Arealität. Tübingen: Niemeyer (Reihe Germanistische Linguistik, Band 66).

Koß, Gerhard (1983): Realisierung von Kasusrelationen in den deutschen Dialekten. In: Besch, Werner et al. (Hrsg.): Dialektologie. 2. Teilband. Berlin/New York: de Gruyter (Handbücher zur Sprach- und Kommunikationswissenschaft, 1.2), 1242-1250.

Polenz, Peter von (1999): Deutsche Sprachgeschichte vom Spätmittelalter bis zur Gegenwart. Band III: 19. und 20. Jahrhundert. Berlin/New York: de Gruyter (de Gruyter Studienbuch).

Schmidt, Jürgen Erich (2017): Vom traditionellen Dialekt zu den modernen deutschen Regionalsprachen. In: Deutsche Akademie für Sprache und Dichtung / Union der deutschen Akademien der Wissenschaften (Hrsg.): Vielfalt und Einheit der deutschen Sprache. Zweiter Bericht zur Lage der deutschen Sprache. Tübingen: Stauffenburg, 105-143.

Zifonun, Gisela (2017): Possessive Attribute. In: Gunkel, Lutz et al.: Grammatik des Deutschen in europäischen Vergleich. 
Das Nominal. Berlin/Boston: De Gruyter (Schriften des Instituts für Deutsche Sprache, 14), 1568-1637.

\section{Internetquelle}

Wasserrab, Jutta (o.J.): Dialektatlas. Eine Deutschlandreise fürs Ohr. URL: https://p.dw.com/p/Hkbv (zuletzt gesehen am 12 . 05. 2021) 